\title{
Resultados Preliminares con Sistemas de Concentración Solar para la Generación de Vapor de Agua
}

\section{Preliminary Results with Solar Concentrating Systems for Water Vapor Generation}

\author{
${ }^{1}$ Carlos Armando Polo Bravo, ${ }^{2}$ Elisban Juani Sacari Sacari, ${ }^{3}$ Jorge Armando Choque Chacolla
}

\begin{abstract}
RESUMEN:
En el presente trabajo experimental se presenta los resultados preliminares obtenidos en el diseño, construcción y evaluación de dos sistemas de concentración solar para la generación de vapor de agua, estos son el Concentrador Solar Cilindrico Parabólico (CCP), y el Reflector Solar Fresnel de Foco Lineal (RESFFOL). La evaluación se ha realizado bajo las condiciones de radiación solar y otros parámetros meteorológicos de la ciudad de Tacna, llegándose a determinar el área eficiente de la superficie reflectante, pérdidas de calor debido al ángulo de inclinación del concentrador, determinación de la eficiencia óptica, determinación del coeficiente global de pérdidas y la determinación de la eficiencia térmica, estos resultados nos indican la posibilidad de usar esta tecnología para el uso de la energía solar y producir vapor de agua a determinada temperatura y presión para múltiples usos, y posteriormente previa investigación para la generación de energía eléctrica.
\end{abstract}

Palabras clave: energía solar, concentración solar, concentrador solar tipo cilíndrico parabólico, concentrador solar tipo Fresnell de foco lineal, vapor de agua

\section{ABSTRACT:}

This experimental work presents the preliminary results obtained in the design, construction and evaluation of two solar concentrating systems for the generation of water steam, these are the Cylindrical Parabolic Solar Concentrator (CPC) and the Solar Fresnel Reflector Focus linear (RESFFOL). The evaluation was carried out under conditions of solar radiation and other meteorological parameters in the city of Tacna,determining an efficient area of the reflecting surface, heat losses due to the angle of inclination of the hub, the optical efficiency determination, determination of the global loss coefficient and the determination of the thermal efficiency, these results indicate the possibility of using this technology for the use of solar energy and produce water steam at certain temperature and pressure for multiple uses, and then, previous the to investigation for electric power generation.

Keywords: solar energy, concentrating energy solar, concentrator parabolic trough solar, concentrator Focus linear solar Fresnell, water vapor

\footnotetext{
'Licenciado en Física. Facultad de Ciencias. Universidad Nacional Jorge Basadre Grohmann

${ }^{2}$ Licenciado en Física Aplicada.

${ }^{3}$ Licenciado en Física Aplicada.
} 


\section{INTRODUCCIÓN}

Actualmente en muchos países desarrollados se cuentan a nivel de producción, comercialización e investigación cuatro grandes sistemas de concentración solar: el sistema Colector Cilíndrico Parabólico (CCP), el sistema Torre Central (STC), el sistema de Reflexión Fresnell de Foco lineal (RESFFOL), y el Sistema Motor Stirling (SMS), los tres primeros sistemas para generar vapor o para fundir sales de alto punto de fusión que son utilizados como fluidos calo portadores para mover una turbina la cual funciona bajo un determinado ciclo termodinámico, que acoplada a un generador eléctrico, generan energía eléctrica gran escala, el cuarto sistema genera energía eléctrica usando como combustible la energia solar concentrada a través de un motor Stirling, estos sistemas se construyen en grandes áreas toda vez que las grandes plantas para la generación de electricidad requieren de grandes áreas de terreno, y en zonas donde se cuenta con un buen recurso energético solar, transforman la energia solar directa a energía eléctrica, los sistemas se muestran en la Figura $\mathrm{N}^{\circ} 01$.

\subsection{Factor de Concentración Solar}

Los lentes o espejos pueden concentrar la radiación solar que atraviesa un área $\mathrm{A}$, denominada abertura del concentrador, sobre un área menor $\mathrm{A}_{c}$, denominada la superficie absorbente, de modo que el factor de concentración X, se define como:

$$
\mathrm{X}=\mathrm{A} / \mathrm{A}_{\mathrm{c}}
$$

Aquí la concentración es definida como el cociente de dos áreas. (Choque 2010, Sacacari, 2008)

Existen otras definiciones, en particular se puede definir la concentración como el cociente entre la irradiancia (la irradiancia es la densidad de la potencia radiante medida en $\mathrm{W} / \mathrm{m}^{2}$ ) en un punto de la superficie absorbente y la irradiación solar en un punto de la abertura del concentrador, esta última concentración, puede variar de sitio en sitio sobre la superficie absorbente.

\subsection{Colector Solar Cilíndrico Parabólico (CCP)}

Un CCP es un sistema que concentra la energía del sol en una línea focal en el cual se encuentra un receptor o tubo absorbente, este cuenta con una cobertor de vidrio para evitar pérdidas de calor al medio ambiente, por el tubo circula agua a un determinado flujo volumétrico, la cual se evapora al pasar a lo largo de la longitud, alcanzando una determinada temperatura y presión, todo el sistema debe de seguir al sol a efectos de que la radiación solar incida perpendicularmente sobre la superficie reflectora (R. Forristal, 2003)

\subsubsection{Partes de un Concentrador Cilindrico Parabólico}

Los elementos principales de un Concentrador Cilíndrico Parabólico se muestran en la siguiente Figura 02, el reflector cilindro parabólico, el tubo absorbente, el sistema de seguimiento del sol, y la estructura metálica.

\section{El Reflector Cilindrico Parabólico}

La función del reflector cilindro parabólico es reflejar y concentrar sobre el tubo absorbente la radiación solar
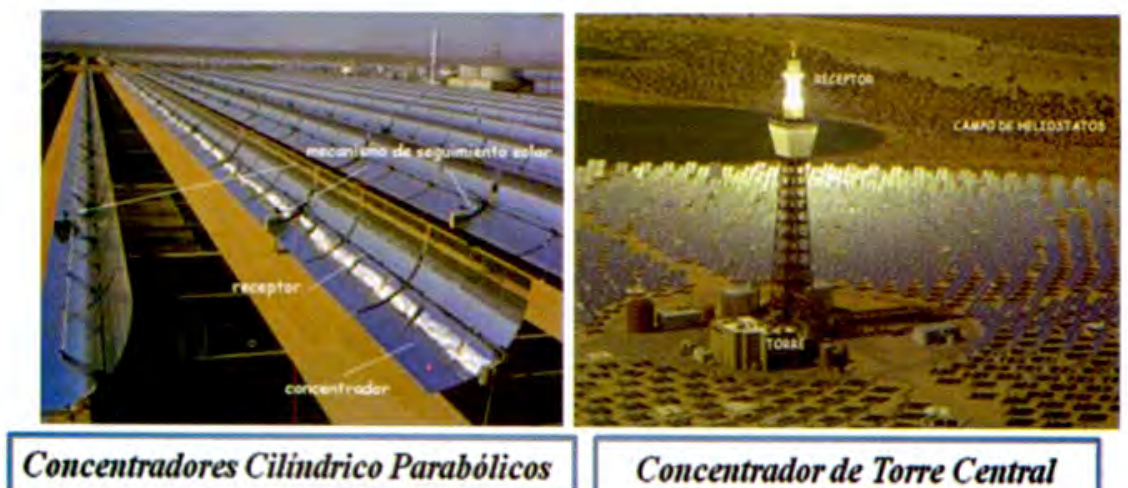

Concentrador de Torre Central

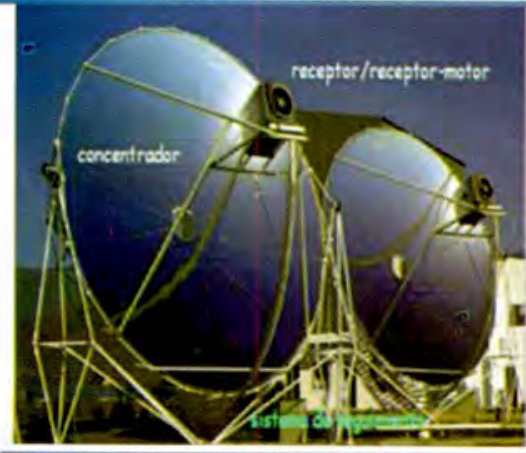

Concentrador Tipo Motor Stirling

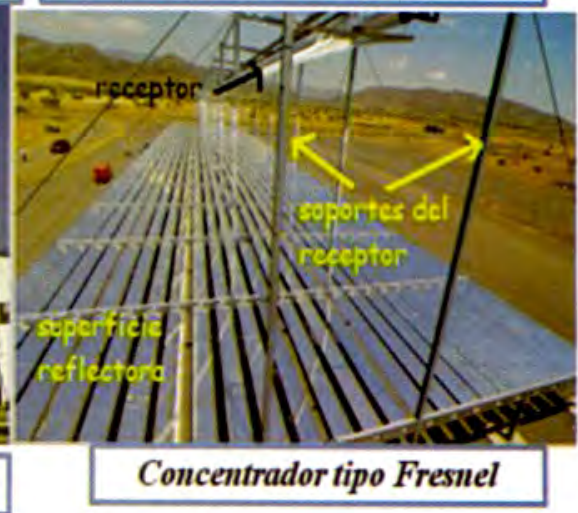

Figura $\mathrm{N}^{\circ} 01$ : Vista general de sistemas de concentración solar actualmente desarrollados Fuente: Solar Paces New, 2003 
directa que incide sobre su superficie. Se trata en definitiva de un espejo curvado en una de sus dimensiones con forma de parábola, que concentra sobre su línea focal toda la radiación solar que atraviesa el plano de abertura. La superficie reflectora generalmente se consigue a base de películas o planchas de plata o aluminio depositadas sobre un soporte que le da la suficiente rigidez. (J.A. Chasseraux, 1990; J.A. Duffi, W.A. Beckman, 1991)

\section{El Tubo Absorbente}

El tubo absorbente es uno de los elementos fundamentales de todo $\mathrm{CCP}$, ya que de él depende en gran medida el rendimiento global del colector, el tubo absorbente de un CCP puede constar de un tubo o, más frecuentemente, de dos tubos concéntricos. En este último caso, el tubo interior, por el que circula el fluido que se calienta, es metálico y el exterior de cristal. El tubo metálico lleva un recubrimiento selectivo que posee una elevada absortividad $(>90 \%)$ y una baja emisividad en el espectro infrarrojo $(<30 \%)$, lo que le proporciona un elevado rendimiento térmico. El tubo de cristal que rodea al tubo interior metálico tiene la doble misión de reducir las pérdidas térmicas por convección en el tubo metálico y de proteger de las inclemencias meteorológicas el recubrimiento selectivo. El tubo de cristal suele llevar también un tratamiento anti reflexivo en sus dos caras, para aumentar su transmisividad a la radiación solar y, consiguientemente, el rendimiento óptico del colector.(Winter C.J. ; Van Hull L.L., 1999)

\section{El Sistema De Seguimiento Solar}

Un CCP, como cualquier sistema solar de

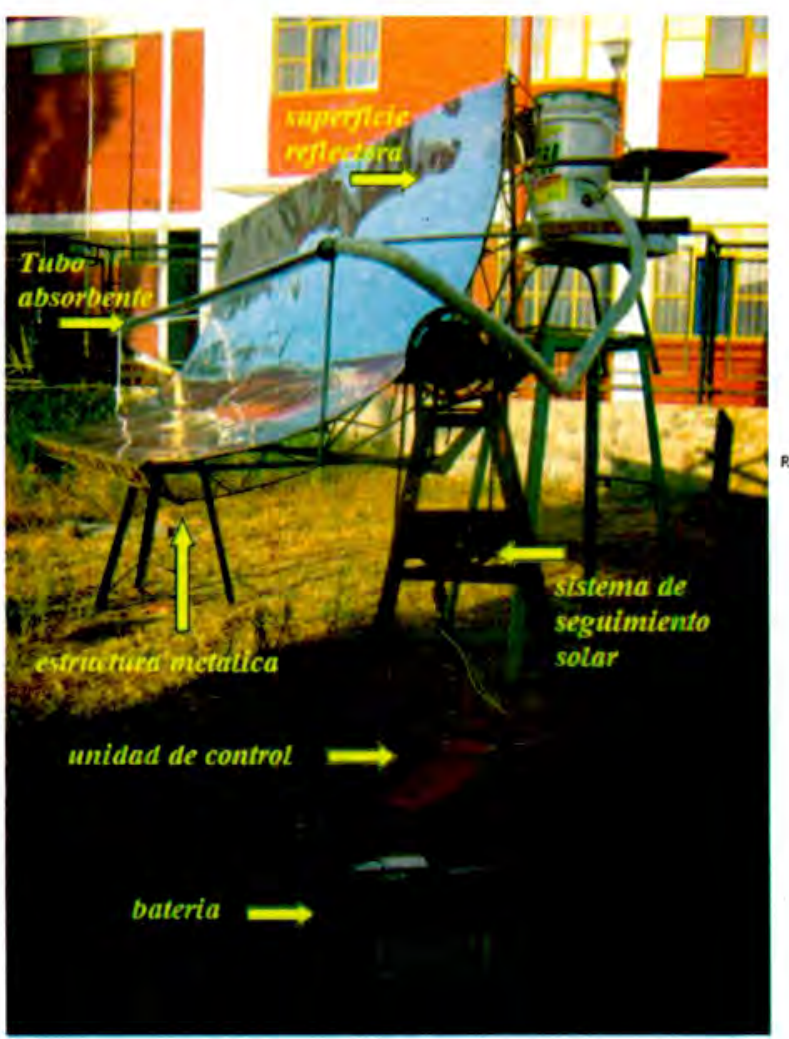

concentración, solo puede aprovechar la radiación solar directa y esto exige que el colector vaya provisto de un mecanismo de seguimiento solar que lo mueva a lo largo del día conforme el sol describe su trayectoria aparente diaria en el cielo. El sistema de seguimiento solar más común consiste en un dispositivo que gira los reflectores cilindro parabólicos del colector alrededor de un eje. La anterior figura derecha muestra esquemáticamente este tipo de seguimiento solar. (Klaib F. Stab, 1992)

\section{La Estructura Metálica}

La misión de la estructura del concentrador es la de dar rigidez al conjunto de elementos que lo componen, a la vez que actúa de interface con la cimentación del colector, otra característica básica deun concentrador solar es su ángulo de aceptación, $\theta_{c}$. En un concentrador tridimensional, todos los rayos solares que inciden en cualquier punto de la abertura A dentro de un cono con abertura angular $2 \theta_{c}$, llegan a la superficie absorbente. En el caso de un concentrador bidimensional, el cono es reemplazado por una cuña de abertura $2 \theta_{C}$ (ver Figura 03 , izquierda). Un rayo que incide con un ángulo mayor que $\theta_{c}$ sobre $A$, eventualmente no es reflejado hacia $A_{c} y$, por lo tanto no es aprovechable.

En general, para cualquier forma de la superficie $A_{c}$ se puede diseñar un concentrador apropiado para lograr la misma concentración posible, considerando que el disco del sol tiene, visto desde la tierra un ángulo $2 \theta_{\mathrm{s}}=32^{\prime}$, la magnitud de la concentración de la radiación solar es limitada.

\section{En este caso nos centraremos en los concentradores}

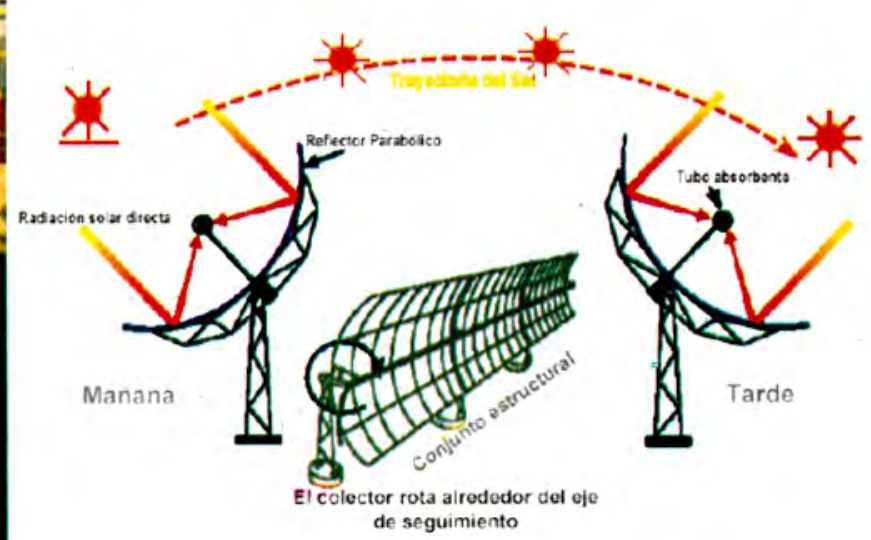

Seguimiento solar tipico de un CCP

Figura $\mathrm{N}^{\circ} 02$ : El CCP y sus partes (izquierda). Sistema de seguimiento solar (derecha) 


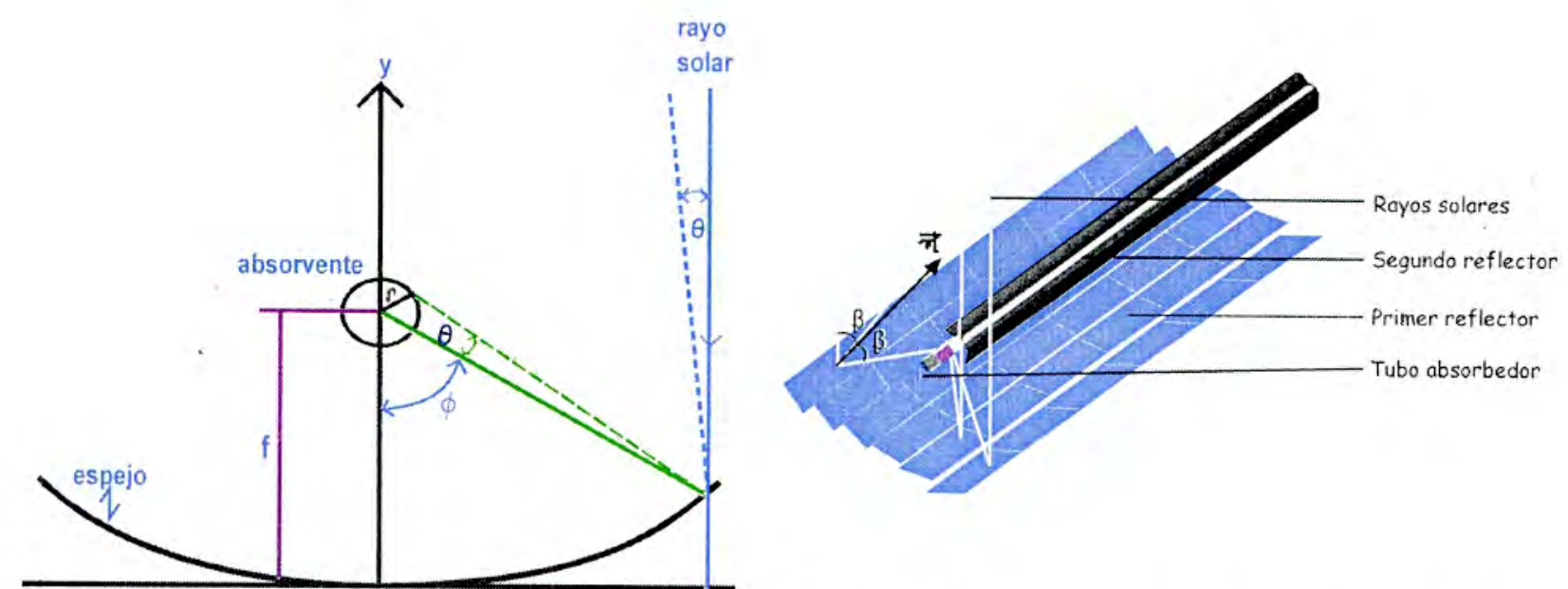

Figura $\mathrm{N}^{\circ} \mathbf{0 3}$ : Ángulo de aceptación en un concentrador CCP (izquierda), esquema de concentrador Fresnel de foco lineal (derecha)

bidimensionales especificamente en los concentradores cilíndricos parabólicos, donde el absorbente es un tubo cilindrico ubicado en el eje focal del concentrador. (A. Meinel, M. Mainel, 1979)

En la Figura 03 izquierda, se muestra un corte tranśversal del Concentrador Cilindrico Parabólico: la parábola esta descrita por la ecuación ( $f$ es la distancia focal):

$$
Y=X^{2} / 4 f
$$

Comparando las ecuaciones se observa que. para un mismo ángulo de aceptación. el CCP tiene una concentración $\pi$-veces inferior a la máxima alcanzable. Estoes una concentracion

$$
X_{0}=213 / \pi=67
$$

Para obtener este valor se debe tener un tubo absorbente con un radio $\mathrm{r}=\mathrm{d} \sin \theta_{s}=0.0046 . \mathrm{d}$.

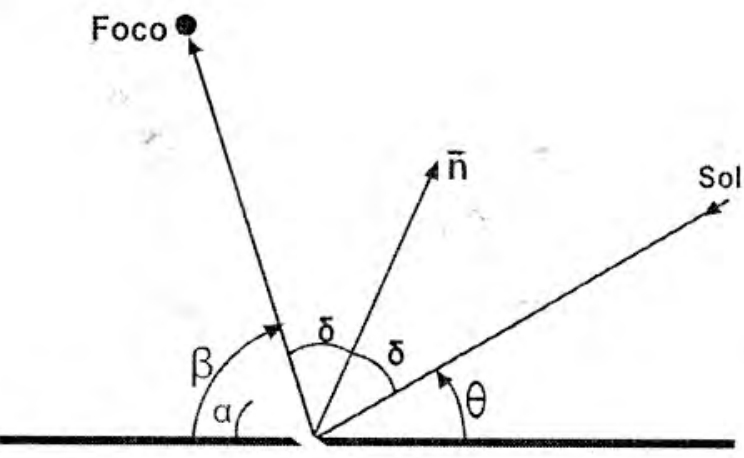

\subsubsection{Reflector Freisnel de Foco Lineal con Segundo Concentrador}

Un reflector Fresnel se genera cortando y formando caras reflectoras en una superficie esencialmente llana. La luz deberá ser reflejada hacia un punto focal o linea focal definida de acuerdo con la ley de Snell, con tal que la normal de la superficie de cada cara reflectora se oriente a cortar en ángulos iguales la luz incidente y el punto focal. En la Figura 04 izquierda se visualiza el principio de funcionamiento de un reflector solar Fresnel de foco lineal ( $\mathrm{H}$. Andreas, Zahler $\mathrm{C}$., Lenchenmuller H.. Meertins M.,Witwer C., Trieb F., Dersch J.)

Este tipo de concentrador puede llevar un segundo concentrador auxiliar para asegurar que los rayos desviados por los reflectores planos (reflector primario) lleguen al tubo absolvedor, tal como se muestra en la Figura 04 derecha. Un concentrador solar solamente trabaja con radiación solar directa, por tal motivo, estos

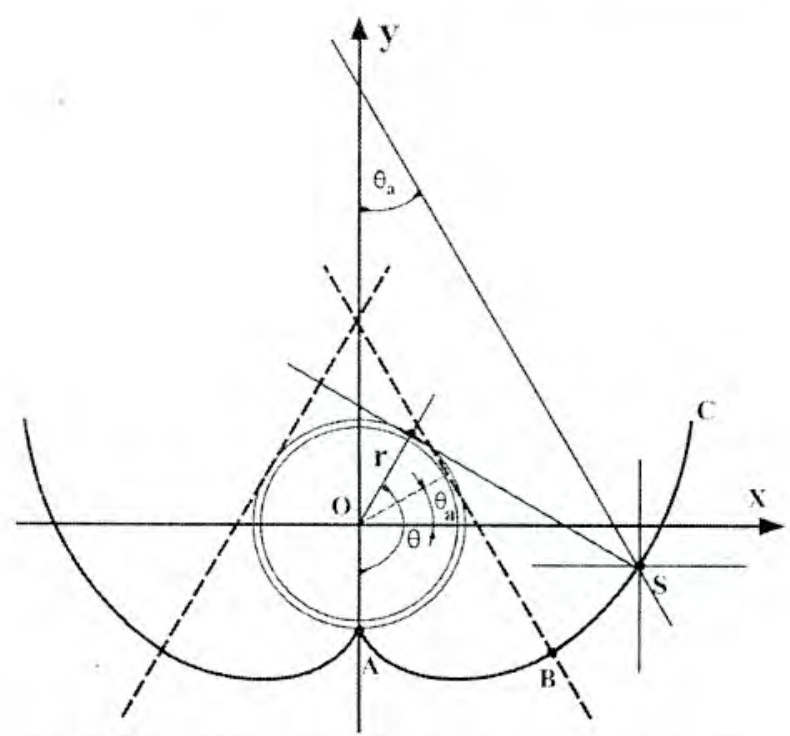

Figura $N^{\circ} 04$ : Esquema de un reflector al incidir un rayo de sol (izquierda). Desarrollo de un concentrador a partir de la envolvente de un círculo de radio del tubo absolvedor (derecha) 
sistemas llevan un sistema de seguimiento solar, la ventaja para el Reflector Solar Fresnel es de usar un sistema de seguimiento del sol de un solo eje, de este a oeste o de norte a sur.

\section{Concentrador Solar de la Envolvente de un Círculo}

Se han experimentado espejos reflectores cilíndricos basados en la evolvente del círculo, en los que por definición, las normales a la evolvente son tangentes al círculo, de forma que todos los rayos incidentes sobre este reflector se reflejan hacia el círculo. (CIEMAT Solar Photo Chemestry Techmology)

RS es tangente en el punto $\mathrm{R}$ del círculo (absolvedor circular lineal). Un importante parámetro para la definición del concentrador es el ángulo de aceptancia $\theta_{a}$, el cual es el rango angular máximo por el cual todos los rayos son aceptados sin necesidad de mover el colector (ver Figura 04 derecha). La solución está dada por dos porciones separadas, una envolvente ordinaria de un círculo desde el punto $\mathrm{A}$ hacia $\mathrm{B}$ y otra porción desde $\mathrm{B}$ hacia $\mathrm{C}$.

Además, el factor de concentración es:

$$
C=\frac{1}{\operatorname{sen} \theta_{c}}=C_{\text {ideal } 2 d}
$$

\section{METODOLOGÍA}

Para el desarrollo de presente trabajo se ha seguido el siguiente procedimiento:

Diseño de los sistemas: para lo cual se ha tenido que revisar las referencias bibliográficas correspondientes, a efectos de conocer los aspectos teóricos y de funcionamiento.

Dimensionamiento: realizado sobre la base de las dimensiones de la plancha de aluminio anodizado, tubo receptor de cobre, vidrio cobertor del tubo absorbente, y material para la estructura de soporte, y sobre el potencial del recurso solar disponible en la región.

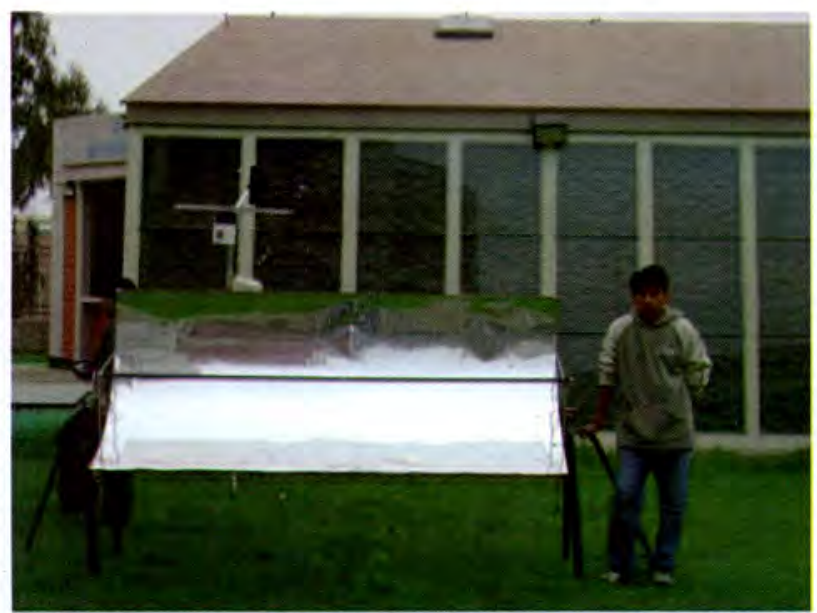

Construcción: sobre la base de los dos primeros pasos, se traza la figura geométrica correspondiente, teniendo mucho cuidado en el trazo, a efectos de minimizar las pérdidas ópticas, y maximizar la concentración solar de los sistemas.

Evaluación: una vez construidos los sistemas se procede a colocar los diferentes sensores para medir temperaturas del fluido de trabajo a lo largo del tubo absorbedor cada $0,80 \mathrm{~m}$, a efectos de la caracterización térmica, temperatura ambiente, irradiancia solar incidente sobre la superficie reflejante, previamente los sistemas se orientan a lo largo del eje en la dirección norte-sur, en forma manual se hace que los sistemas sigan al sol, la evaluación se realiza con y sin líquido de trabajo, determinando finalmente las curvas de temperatura, eficiencia de los sistemas y el factor de concentración.

Procesamiento da datos: con los datos experimentales obtenidos, y los parámetros dimensionales y ópticos de los sistemas se realizan los cálculos correspondientes.

\section{RESULTADOS EXPERIMENTALES}

\subsection{Resultados con el Concentrador Cilíndrico Parabólico}

\subsubsection{Características del Concentrador Cilindrico Parabólico}

El concentrador cilindrico parabólico evaluado tiene las siguientes características: (ver Figura 05)

La superficie reflectante es de plancha de aluminio anodizado de alto coeficiente de reflexión, de $0.2 \mathrm{~mm}$ de espesor, el receptor o tubo absorbente es de aluminio, pintado con pintura negro mate para aumentar la capacidad de absorción de calor, tiene un radio de 1.27 $\mathrm{cm}$, el área de abertura del CCP es de $2.662 \mathrm{~m}^{2}$, para el cobertor del tubo absorbente se utilizó tubos de vidrio procedente de tubos fluorescentes malogrados (ver Figura 06 derecha) y colocadas concéntricamente al tubo absorbente para evitar pérdidas de calor por convección y radiación al medio ambiente.

Figura $\mathbf{N}^{\circ} 05$ : Vista Frontal del Concentrador Cilíndrico Parabólico diseñado, construido y evaluado (izquierda); Tubos fluorescentes malogrados, reutilizados como cobertor del tubo absorbente y tubo absorbente de aluminio con el cobertor de vidrio, y junta de unión entre tubos de vidrio (derecha) 


\subsubsection{Factor deconcentración}

El factor de concentración teórico, se determina con la ecuación siguiente:

$$
X=\frac{A}{I_{i}}=\frac{2 d L}{2 \pi r L}=\frac{\text { 国 }}{\pi r}=\frac{55 \mathrm{~cm}}{3.1416 \times 1.27 \mathrm{~cm}}=13.78
$$

\subsubsection{Area Eficiente de la Suprerficie Reflectanto}

Sabiendo que las medidas del CCP son:

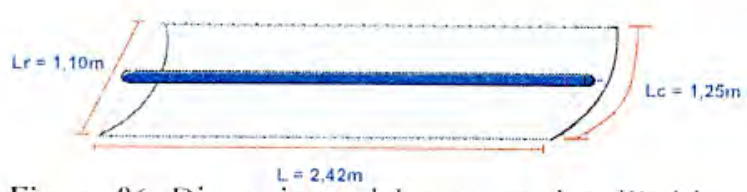

Figura 06: Dimensiones del concentrador cilindrico parabólico

Elarea reflectante es: $A k=L \times L$

Dónde: $A_{4}$ el área de la superficie reflectante $\left[\mathrm{m}^{2}\right], \mathrm{L}$, es la longitud del lado curvo [m]. Lr es la: longitud del lado recto $[\mathrm{m}]$. y $\mathrm{L}$ es la longitud del concentrador $[\mathrm{m}]$. Entonces tenemos que $\mathbf{A}_{\mathrm{k}}=\mathbf{3 . 0 2 5 \mathrm { m } ^ { 2 }}$

El $12 \%$ del área reflectante $\left(0.363 \mathrm{~m}^{2}\right)$ es deficiente debido a deformaciones en toda la superficie reflectante (ver partes amarillas en la Figura 07 ), adicionándole un $5.45 \%$ de superficie reflectante $\left(0.1648 \mathrm{~m}^{2}\right)$ que se pierde debido al ángulo de incidencia de la radiación solar mayor o menor a la normal del concentrador cilindrico parabólico, haciendo un total de un $17.45 \%$ de area reflectante que se pierde $\left(0.5278 \mathrm{~m}^{2}\right)$ debido a estos dos tipos de perdidas ópticas, quedándonos un área de trabajo de $2.497 \mathrm{Im}^{2}(82.55 \%$ del área total del concentrador).

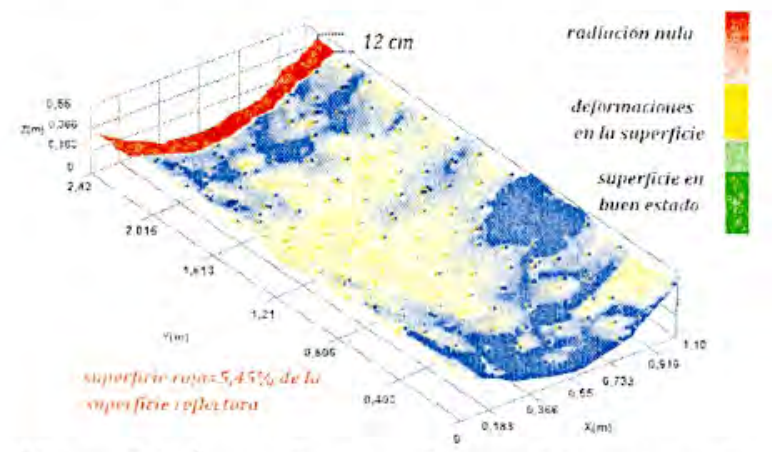

Figura 07: Área reflectora eficiente del concentrador cilindrico parabólico, pérdidas ópticas y pérdidas por el ángulo de incidencia de la radiación solar

Para las siguientes aplicaciones deberemos tomar en cuenta la siguiente relación:

$\frac{A \text { abertura }}{A_{R}}=\frac{L_{R} \times L}{L_{r} \times L}=\frac{L_{R}}{L_{i}}=0.88 ; A_{\text {abertura }}=0.88 . A_{R}$

Siendo A ol área de abertura del concentrador cilindrico parabólico. Con este resultado podemos calcular ahora el área de abertura eficiente del concentrador cilindrico parabólico, esto es:

$$
\begin{aligned}
& A_{A}=0.88\left(A_{H}-17.45 A_{H}\right) \\
& A_{\text {a wermonatianth }}=2.197 \mathrm{~m}^{2}
\end{aligned}
$$

\section{1.t. Cáloulo de la eficiencia óptica}

En las tablas siguientes se muestran los resultados obtenidos experimentalmente de la eficiencia óptica del CCP. con y $\sin$ tubo de vidrio cobertor del tubo absorbente.

Tabla 01: Tubo absorbente con el Cobertor Vidrio

\begin{tabular}{lc}
\hline TUBO ABSORBENTE CON EL COBERTOR \\
VIDRIO \\
\hline Temperatura de entrada $\mathrm{T}_{\mathrm{e}}\left({ }^{\circ} \mathrm{C}\right)$ & 22.70 \\
Temperatura de salida $\mathrm{T}_{\mathrm{s}}\left({ }^{\circ} \mathrm{C}\right)$ & 25.30 \\
Temperatura ambiente $\mathrm{T}_{\mathrm{amb}}\left({ }^{\circ} \mathrm{C}\right)$ & 24.00 \\
Radiación Solar $\mathrm{G}_{\mathrm{t}}\left(\mathrm{W} / \mathrm{m}^{2}\right)$ & 973.78 \\
Calor especifico del agua $\mathrm{c}\left(\mathrm{kJ} / \mathrm{kg}{ }^{\circ} \mathrm{C}\right)$ & 4.19 \\
Área del tubo absorbente $\left(\mathrm{m}^{2}\right)$ & 0.50 \\
Flujo de agua $\mathrm{m}(\mathrm{kg} / \mathrm{s})$ & 0.0316 \\
Eficiencia óptica $\boldsymbol{\alpha} \tau$ & $\mathbf{0 . 7 0 6 3 6 4 4}$ \\
\hline
\end{tabular}

Tabla 02: Tubo absorbente $\sin$ el Cobertor Vidrio

TUBO ABSORBENTE SIN EL COBERTOR VIDRIO

\begin{tabular}{lc}
\hline Temperatura de entrada $\mathrm{T}_{\mathrm{e}}\left({ }^{\circ} \mathrm{C}\right)$ & 23.00 \\
Temperatura de salida $\mathrm{T}_{\mathrm{s}}\left({ }^{\circ} \mathrm{C}\right)$ & 24.80 \\
Temperatura ambiente $\mathrm{T}_{\text {amb }}\left({ }^{\circ} \mathrm{C}\right)$ & 23.90 \\
Radiación Solar $\mathrm{G}_{\mathrm{t}}\left(\mathrm{W} / \mathrm{m}^{2}\right)$ & 801.49 \\
Calor específico del agua $\mathrm{c}\left(\mathrm{kJ} / \mathrm{kg}{ }^{\circ} \mathrm{C}\right)$ & 4.19 \\
Área del tubo absorbente $\left(\mathrm{m}^{2}\right)$ & 0.50 \\
Flujo de agua $\mathrm{m}(\mathrm{kg} / \mathrm{s})$ & 0.0294 \\
Eficiencia óptica $\boldsymbol{\alpha \tau}$ & $\mathbf{0 . 5 5 2 7 7 8 3}$ \\
\hline
\end{tabular}

\subsubsection{Cálcuto del Coeficiente Global de Pérdidas}

En las siguientes tablas se muestran los valores del coeficiente global de pérdidas, obtenidos con los datos experimentales correspondientes:

Ver tabla $\mathrm{N} 03$ 
Tabla 03: Coeficiente Global de pérdidas para el tubo absorbente con el cobertor de vidrio

\section{COEFICIENTE GLOBAL DE PERDIDAS \\ PARA EL TUBO ABSORBENTE CON EL COBERTOR VIDRIO}

\begin{tabular}{lc}
\hline Temperatura de entrada $\mathrm{T}_{\mathrm{e}}\left({ }^{\circ} \mathrm{C}\right)$ & 69.81 \\
Temperatura de salida $\mathrm{T}_{\mathrm{s}}\left({ }^{\circ} \mathrm{C}\right)$ & 69.35 \\
Temperatura media $\mathrm{Tm}\left({ }^{\circ} \mathrm{C}\right)$ & 69.58 \\
Temperatura ambiente $\mathrm{T}_{\text {amb }}\left({ }^{\circ} \mathrm{C}\right)$ & 19.19 \\
Calor especifico del agua $\mathrm{c}\left(\mathrm{kJ} / \mathrm{kg}{ }^{\circ} \mathrm{C}\right)$ & 4.19 \\
Flujo de agua $\mathrm{m}(\mathrm{kg} / \mathrm{s})$ & 0.083 \\
Área del tubo absorbente $\left(\mathrm{m}^{2}\right)$ & 0.1931 \\
$\mathrm{~A}_{\text {tubo absorbente }}\left(\mathrm{m}^{2}\right)$ & $\mathbf{1 6 . 4 2 5 1}$ \\
$\mathrm{U}_{\mathrm{L}}\left(\mathrm{W} / \mathrm{m}^{2}{ }^{\circ} \mathrm{C}\right)$ & \\
\hline
\end{tabular}

Tabla 04: Coeficiente Global de pérdidas para el tubo absorbente sin el cobertor de vidrio

COEFICIENTE GLOBAL DE PERDIDAS

PARA EL TUBO ABSORBENTE SIN EL COBERTOR VIDRIO

\begin{tabular}{lc}
\hline Temperatura de entrada $\mathrm{T}_{\mathrm{e}}\left({ }^{\circ} \mathrm{C}\right)$ & 68.65 \\
Temperatura de salida $\mathrm{T}_{\mathrm{s}}\left({ }^{\circ} \mathrm{C}\right)$ & 67.82 \\
Temperatura media $\mathrm{Tm}\left({ }^{\circ} \mathrm{C}\right)$ & 68.235 \\
Temperatura ambiente $\mathrm{T}_{\text {amb }}\left({ }^{\circ} \mathrm{C}\right)$ & 20.29 \\
Calor especifico del agua $\mathrm{c}\left(\mathrm{kJ} / \mathrm{kg}{ }^{\circ} \mathrm{C}\right)$ & 4.19 \\
Flujo de agua $\mathrm{m}(\mathrm{kg} / \mathrm{s})$ & 0.083 \\
Área del tubo absorbente $\left(\mathrm{m}^{2}\right)$ & 0.1931 \\
$\mathrm{~A}_{\text {tubo absorbente }}\left(\mathrm{m}^{2}\right)$ & $\mathbf{3 1 . 1 4 7 9 7 5}$ \\
$\mathbf{U}_{\mathrm{L}}\left(\mathrm{W} / \mathrm{m}^{2}{ }^{\circ} \mathrm{C}\right)$ & \\
\hline
\end{tabular}

\subsubsection{Determinación de la Eficiencia Térmica.}

Una vez determinadas la eficiencia óptica y el coeficiente global de pérdidas, partimos de la ecuación.

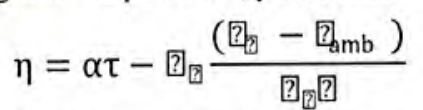

$\mathrm{Y}$; el factor de concentración $(X)$, reemplazando el área de abertura $\left(A_{\text {athertura }}\right)$ por el área de abertura eficiente $\left(A_{\text {uberiur efficente }}\right)$ que es igual a $2.197 \mathrm{~m}^{2}$, entonces tenemos que el nuevo factor de concentración será:

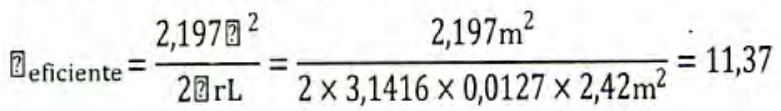

Para el concentrador con tubo absorbente con el cobertor de vidrio.(C. Polo, H. Torres, 2005)

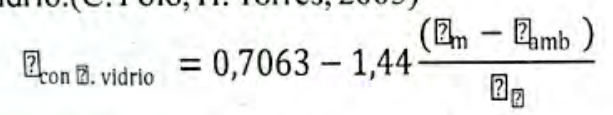

Comparando las eficiencias térmicas para los casos donde el tubo absorbente cuenta con el cobertor de vidrio y sin el cobertor de vidrio tenemos:

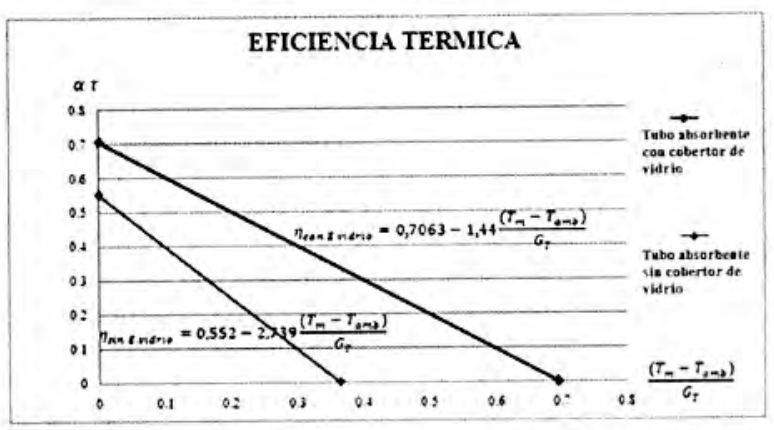

Figura 08: Comparación de la eficiencia térmica del concentrador cilíndrico parabólico para los casos donde el tubo absorbente esta con y sin el cobertor de vidrio

\subsection{Resultados con el Concentrador Fresnel de Foco Lineal con Segundo Concentrador}

El esquema funcional del sistema concentrador Fresnel de foco lineal y con segundo concentrador (envolvente del círculo), diseñado, construido y evaluados, en vista de frente y de costado se muestra en la Figura 09.

En reflectores Fresnel hay que considerar para su diseño que los reflectores no se hagan sombra, para lo cual se ha decidido poner una separación de $10 \mathrm{~cm}$. entre cada reflector. (J. Choque $\mathrm{Ch}, 2011$ ). El área total de colección de la radiación solar es de 1.90 metros de ancho por 2.9 metros de largo conformados por 10 filas de reflectores de material espejo de vidrio con un ancho de $10 \mathrm{~cm}$. cada uno. Es decir el área efectiva del colector de reflectores Fresnel del $50 \%$ del área total.

Se usaron 20 espejos con las dimensiones de $0.1 \mathrm{~m}$ de ancho con $1.44 \mathrm{~m}$ de largo las cuales fueron ajustadas con silicona en los 10 soportes individuales, dos espejos por cada soporte.

El segundo concentrador (Figura 10) se ha diseñado pensando en usar como reflectores plancha de aluminio anodizado tipo especular (reflejante) de $3 \mathrm{~mm}$ de espesor y como absolvedor una tubería de cobre pintada de color negro mate de 3 metros de largo y de diámetro I"; para desarrollar la curva característica del segundo concentrador se usó el diámetro externo del tubo de cobre con un radio de $1.43 \mathrm{~cm}$.

El material aislante para este segundo concentrador fue lana de vidrio. La estructura de soporte está constituida de perfiles de ángulo de fierro negro de 1" $\times 2.5 \mathrm{~mm}$ y como cobertor vidrio semidoble y la tapa superior de plancha plana galvanizada de $3 / 32$ " de espesor.

\subsubsection{Determinación del Factor de Concentración}

Es preciso indicar que el área absolvedora del colector de reflectores Fresnel es el área de abertura del segundo concentrador. Para esto existe dos factores de concentración, la del campo de reflectores Fresnel y otra para el segundo concentrador. Para conocer el factor de concentración total se obtiene del producto de cada concentrador. 


$$
C_{\text {total }} \equiv C_{\text {leF }=\text { freshel }} \times C_{\text {2ido_etente }}
$$

它 faetor de eoneentraelón del primer eoneentrador = refleetor Fresnel es dado por:

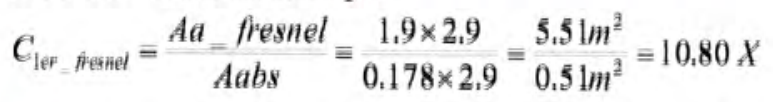

Donde $\mathrm{A}_{\mathrm{a} \text { tessel }}=$ área de abertura del reflector Fresnel, $\mathrm{y}$ $\mathrm{A}_{\text {abs }} \equiv$ área absolvedor, para este easo es el área de abertura del segundo concentrador. Este resultado $\mathrm{C}_{\text {lef_fresed }}=10,80 \mathrm{X}$ se ha considerado el área total con área reflectiva más área en blanco. Considerando que el área reflejante es el $\mathbf{5 0 \%}$ de toda el área de apertura del primer concentrador tenemos:

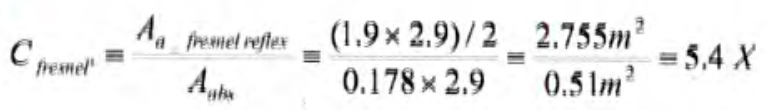

Para el segundo concentrador el factor de concentración C ideal para el ángulo de aceptancia igual a $22,5^{\circ}$ es 2.61X, pero como se ha cortado parte de la curva original para que esta puede aceptar toda la radiación reflejada por el área de reflectores Fresnel se tiene el

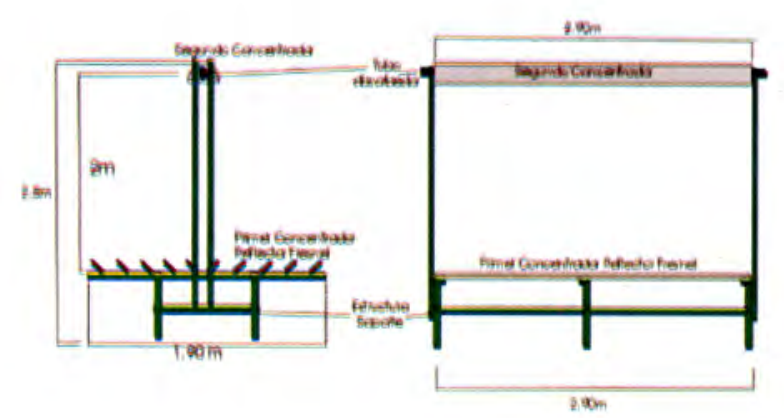

nuevo valö:

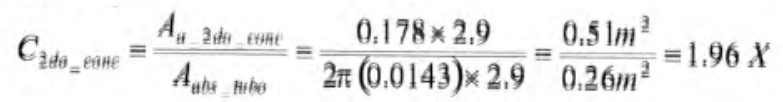

El factor de coneentración total del sistema eolector de reflectores Fresnel y segundo concentrador reemplazando en la eeuación de factor de concentración tenemos:

$$
C_{\text {total }} \equiv C_{\text {fieshel }} \times C_{\text {2do_enteen }} \equiv 10.58 x
$$

\subsubsection{Curva de Calentamiento del Tubo Absorbedor}

Para determinar la máxima temperatura que aleanza el tubo absolvedor en vaeio $y$ el tiempo que tomara aleanzarlo, la disposición experimental del equipo se muestra en la figura. 11 izquierda, para esto se han colocado seis termocuplas dispuestas en el interior de la pared del tubo absolvedor a lo largo del tubo, la prueba eonsiste en el monitoreo de la temperatura con respecto al tiempo. La estruetura del concentrador fue orientada hacia el norte y los reflectores seguian al sol de este a oeste.

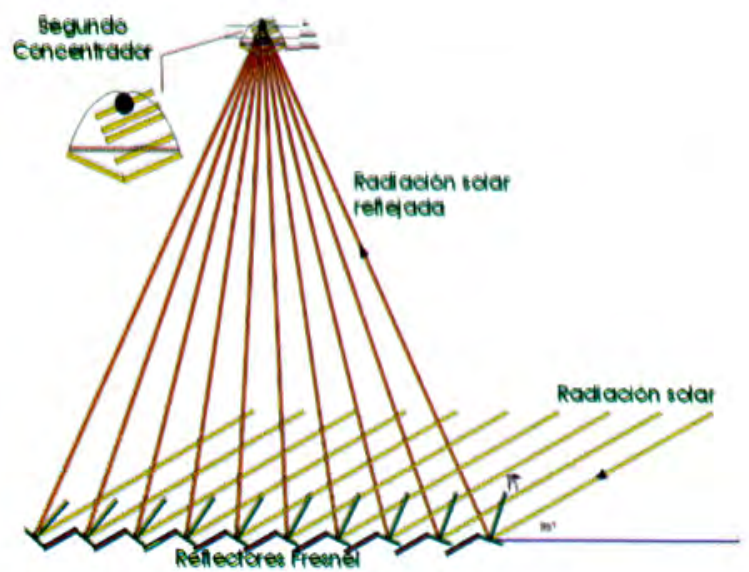

Figura $\mathrm{N}^{\circ} 09$ : Vista general de frente y costado del concentrador solar Fresnell de foco lineal con segundo concentrador (izquierda); esquema de funcionamiento reflexión-concentración de la radiación solar del sistema
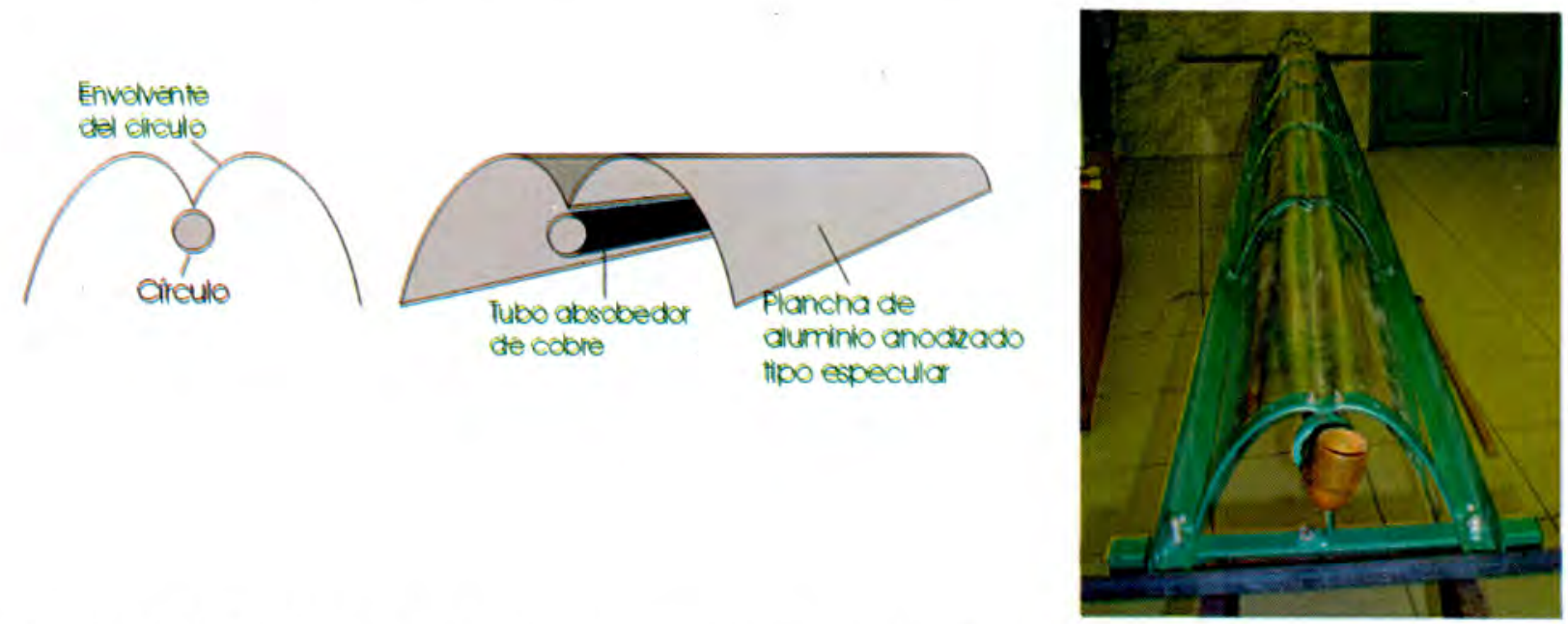

Figura $N^{\circ} 10$ : Vista del diseño del segundo concentrador (izquierda); vista longitudinal del segundo concentrador construido y materiales usados (derecha) 

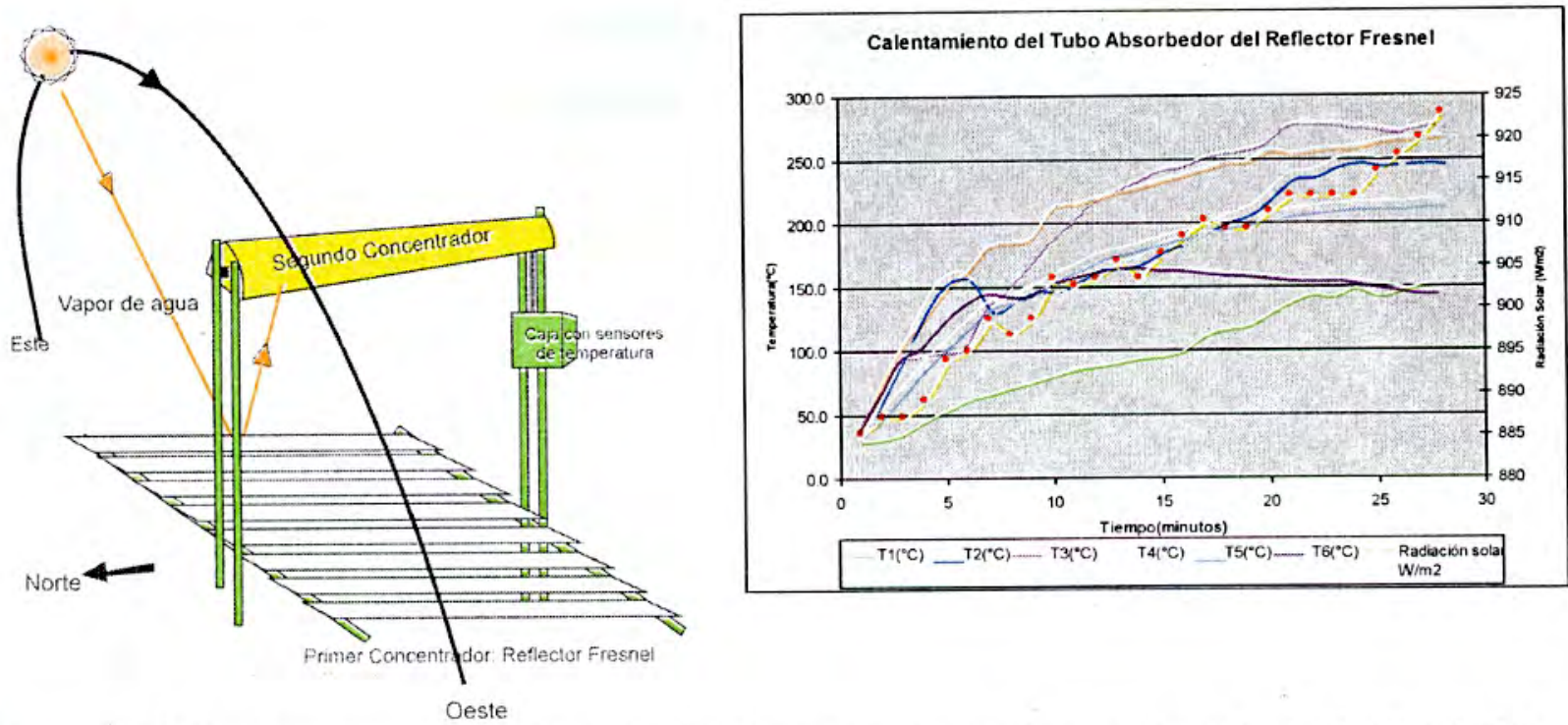

Figura $\mathrm{N}^{\circ} 11$ : Esquema experimental para determinar la curva de calentamiento del tubo absolvedor (izquierda); curva de calentamiento a lo largo del tubo absolvedor (derecha)

\subsubsection{Determinación de la eficiencia óptica ( $\tau \alpha)$ yel coeficiente global de pérdidas:}

Los valores determinados experimentalmente para este sistema en lo referente a la eficiencia óptica es de $0.49, \mathrm{y}$ para el coeficiente global de pérdidas térmica de 23.8 $\mathrm{W} / \mathrm{m}^{2 \circ} \mathrm{C}$.

\subsubsection{Determinación de la Eficiencia Térmica y Curva de Calentamiento}

Con la determinación de la eficiencia óptica y el coeficiente global de pérdidas obtenemos la ecuación de la eficiencia térmica. considerando que $C_{\text {zat ato }}=A_{\text {therror }}$ $A_{\text {intulintas }} y$ además sabemos que:

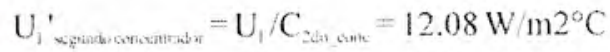

Comparación de Eficiencia Térmica

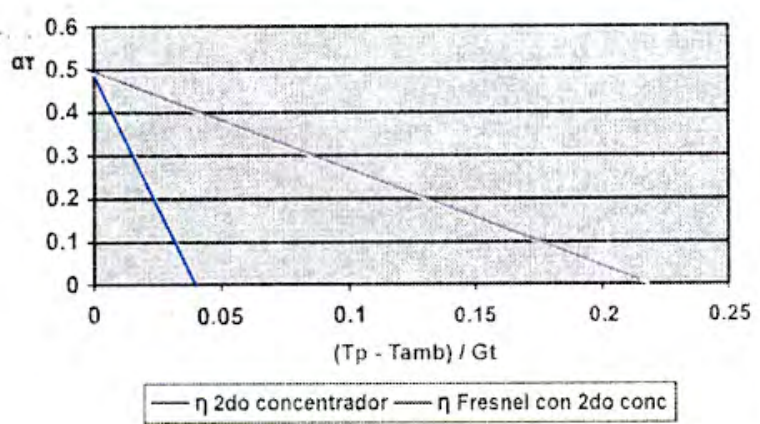

Considerando la radiación solar directa reflejada desde el primer concentrador y la concentración Ctotal = $10.58 \mathrm{X}$

$\eta=0.49-2.24\left(\mathrm{~T}_{n}-\mathrm{T}_{\text {, n }}\right) / \mathrm{G}_{\mathrm{i}}$

La ecuación anterior representa la eficiencia térmica del concentrador solar Fresnel con el segundo concentrador de foco lineal en condiciones de concentración solar para obtener una curva a condiciones operativas $T_{n}, T_{\text {amb }}$. $\mathrm{y} \mathrm{G}_{\text {. }}$

\section{DISCUSIÓN DE RESULTADOS:}

Analizando los resultados obtenidos con el sistema de concentración solar Cilíndrico Parabólico, observamos que colocando el vidrio tubular cobertor del tubo absorbente de dismintyen fuertemente las pérdidas térmicas hacia el medio exterior, tal como puede

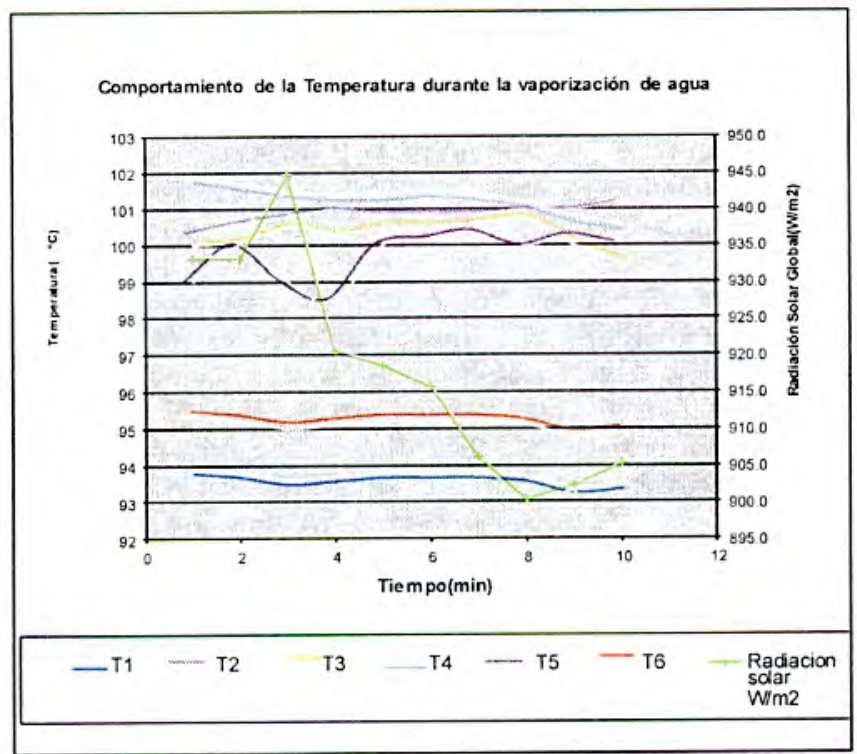

Figura $\mathrm{N}^{\circ}$ 12: Curvas comparativas de la eficiencia térmica entre el concentrador Fresnel con segundo concentrador. y la del segundo concentrador (derecha): comportamiento de la temperatura del agua y vapor de agua en el segundo concentrador (derecha) 
verificarse en los cuadros de resultados correspondientes, por otro lado considerando la orientación del colector sobre el plano horizontal, $y$ el movimiento diario de la tierra sobre su propio eje, las pérdidas por incidencia de la radiación solar sobre el colector es apreciable considerando que el colector y el absorbedor deben seguir continuamente al sol, también es importante la calidad de la superficie reflejante porque influye en la reflexión de la radiación solar sobre el tubo receptor, el sistema produce vapor de agua a una temperatura de $100{ }^{\circ} \mathrm{C}$. Respecto a los resultados obtenidos con el sistema Fresnell de Foco Lineal el mecanismo de seguimiento del sol lo realiza las placas planas de espejos reflejante. los cuales reflejan la radiación solar directa incidente sobre el segundo concentrador de doble envolvente, se ha obtenido vapor de agua con temperatura de $110^{\circ} \mathrm{C}$. se observa los fenómenos de pérdida de área reflectora por efecto de la dirección incidente de la radiación solar. El hecho de que nuestros estudiantes hayan llegado a diseñar, construir y evaluar los sistemas indicados, nos muestra la gran posibilidad de diseñar sistemas muchos más grandes para generar vapor de agua, y desde luego energia eléctrica, ideas basadas en hechos y con tecnologia limpia amigable con nuestro ambiente.

\section{CONCLUSIONES:}

En una primera etapa se ha diseñado, construido, y evaluado dos sistemas de concentración solar para la generación de vapor de agua; el sistema Concentrador Cilindrico Parabólico (CCP), y el Concentrador Fresnel de Foco Lineal con segundo concentrador, los principales resultados experimentales se muestran en el desarrollo del paper, con ambos sistemas se han obtenido vapor de agua a temperatura de $100{ }^{\circ} \mathrm{C}$ y a presión atmosférica, los cuales nos indican la factibilidad de la construcción de sistemas de mayor dimensión usando materiales de fabricación nacional. y sobre todo se ha comprobado la funcionalidad de los sistemas.

Igualmente se ha desarrollado toda una metodología y Know ledge para la evaluación experimental básica de estos sistemas usando instrumentos y equipos que cuente el CERT de la UNJBG, para posteriores y mejores evaluaciones se deben de adquirir equipos modernos de acuerdo al campo de utilización de la energía solar concentrada. El desarrollo de estas tecnologias sumada al gran potencial energético solar de la región Tacna, es una gran altemativa para solucionar la oferta de agua para uso poblacional. industrial y agrícola. y para la generación de energía eléctrica a gran escala, usando el desierto sosteniblemente, y para múltiples procesos industriales.

Para que los sistemas funcionen automáticamente es necesario desarrollar sistemas de seguimiento del sol, y para una mejor aplicabilidad de los sistemas CCP y RESFFOL, debe de diseñarse, construirse y evaluarse sistemas de mayor dimensión a efectos de obtener vapor de agua a mayor temperatura y presión.

\section{REFERENCIAS BIBLIOGRÁFICAS}

Choque Chacolla, Jorge, Diseño, Construcción y Evaluación de un Concentrador Solar Fresnell de Foco Lineal, Trabajo de Tesis. 2011, UNJBG, Tacna, Perú.

Franz Trieb, Solar Electricity Generación, DLR-Institut fur Technische Thermodynamik-Verlag. Alemania, 1995.

Jhon A, Duffi, William A, Beckman, Solar Engineering of Thermal Processes, Segunda Edición. Jhon Wiley \& Sons, USA, 1991.

J.A. Chasseriaux, Conversión Térmica de la Radiación Solar, Libreria Agropecuaria S.A., Primera Edición, Argentina, 1990.

Klaib, F. Staib, Solar Thermal Power Plants for the Mediterranean Area, Springer-Verlag, Alemania, 1992.

Manrique J.A., Energía Solar, Fundamentos y Aplicaciones Fototérmicas, Editorial Harla S.A., 1984, México.

Meinel Aden B. \& P Meinel Marjorie, APPLIED SOLAR ENERGY, Addison - Wesley Publishing Company Inc. 1976, EEUU.

Polo Bravo, Carlos A.; Torres Muro Hugo A., Métodos Experimentales para la Evaluación de Colectores y Termas Solares, Centro de Energias Renovables de Tacna (CERT) - Facultad de Ciencias - Universidad Nacional Jorge Basadre Grohmann de Tacna, 2005 Perú

R. Forristall: Heat Transfer Analisys and Modeling of a parabolic trough Solar Receiver Implemented in Engineering Equation Solver; National Renewable Energy Laboratory, Octubre 2003: Colorado - EEUU.

Sacari Sacari, Elisban: Díseño, Construcción y Evaluación de un Concentrador Cilíndrico Parabólico, Informe de Prácticas Pre Profesionales, 2008, UNJBG, Tacna. Perú.

Servicio Nacional de Meteorologia e Hidrología (SENAMHI) en convenio con la dirección Ejecutiva de Proyectos del Ministerio de Energia y Minas (DEPMINEM). Atlas de Energía Solar del Perú, Edición 2003 -Perú

Winter C. J., Sizmann R.L., Vant Hull L.L. (Eds.); Solar Power Plants, Springer-Verlag, 1991, EEUU.

Centro de Investigaciones Energéticas. Medioambientales y Tecnológicas - CIEMAT, Solar PhotoCHemestry Technology, Plataforma Solar de Almeria

www.psa.es/webeng/areas/quimica/docs/solar photoc hemistry technology.pdf

Fernández Diez Pedro, Colectores de Concentración de media temperatura, 
www.personales.ya.com/universal/TermoWeb/Energia sAlternativas/solar/PDFs/Solar4.pdf

Häberle Andreas, Zahler Christian, Lerchenmuller Hansjorg, Meertins Max, Wittwer Christof, Trieb Franz, Dersch Jurgen; The Solarmundo Line Focussing Fresnel Collector-Optical and Thermal Performance and Cost Calculations.

www.pse.de/downloads/fresnel.solarpaces.pdf
Solar Paces New, Issue Fourteen: March 2003 (PAPER) www.solarpaces.org/sp 14f.pdf

\section{Correspondencia:}

Carlos Armando Polo Bravo

Centro de Energías Renovables de Tacna (CERT)

Av. Miraflores s $/ \mathrm{n}$. Tacna. Perú

polobravocarlos@yahoo.es 